\title{
Ciguri. Voyage(s) au pays des Tarahumaras
}

RAYMONDE CARASCO

Toulouse

1. INTRODUCTION: LE PROBLEME D'ÉCRIRE UN SCÉNARIO, ENTRE CINÉMA ET ETHNOGRAPHIE

Il est extrêmement difficile d'écrire un scénario d'Anthropologie visuelle, dans le cas où il s'agit de réaliser un véritable film ethnographique, c'està-dire un film ayant sa propre autonomie, où seules les images visuelles et sonores donnent à voir et à entendre, hors logos, hors tout commentaire discursif, non seulement le matériau ethnographique recueilli, mais aussi (et surtout) l'idée du film, le sens, nécessairement invisible, de l'événement présenté. Il s'agit donc d'inventer une parole-cinéma, de construire une pensée-cinéma. Par la mise en cinéma, le montage.

L'anthropologue entre alors dans un devenir-cinéaste: l'ethnographie est alors l'affirmation de la puissance des signes, hors commentaire ethnologique, comme il a été dit. En ce sens, il est vrai, notre propre trajet fut inverse: c'est d'abord en tant que cinéaste, dans un projet singulier, entre littérature et cinéma, cinéma et ethnologie (aller sur les traces du Voyage qu'Antonin Artaud fit en 1936 au Mexique, au Pays des Tarahumaras) que nous sommes entrée, comme malgré nous, à travers une recherche de vingt années (1977-1998) dans un devenir-ethnographe. Il y aurait donc une sorte de va-et-vient, un double chemin, entre deux processus: devenir-cinéaste (de l'ethnographe), devenir-ethnographe (du cinéaste).

Le problème d'un véritable film ethnographique réside alors en ceci: il ne saurait s'agir de pure fiction, c'est la réalité ethnographique qui commande, une réalité à tous égards périlleuse et risquée, liée au hasard comme à la nécessité d'un événement par essence inattendu, imprévisible et qu'il faut capter comme s'il avait lieu une seule et unique fois — c'est selon notre expérience, la condition d'un document où la présence blanche n'introduit pas trop d'effets de représentation secondaires, laisse le rite initiatique, ses exécutants et spectateurs, dans la rigueur et le sacré d'une dimension collective. 
Le principe fondateur d'un film ethnographique pourrait s'énoncer ainsi: se soumettre à "la cruelle exigence du réel" (Artaud), au surgissement du nouveau, laisser advenir l'événement. La question est alors celle-ci: quel piège, quel dispositif, quel agencement cinématographique créer pour capter (capturer) l'événement? C'est ce problème que l'écriture du scénario doit poser, selon des règles chaque fois à inventer, en fonction de la singularité de chaque projet.

\section{IDÉE DU FILM: CIGURI OU L'EXPERIENCE TARAHUMARA}

Ce film se propose de montrer les Rites du Ciguri chez les Indiens Tarahumaras, au Mexique, aujourd'hui, à partir des textes qu'Antonin Artaud n'a cessé d'écrire et de ré-écrire sur les Tarahumaras ${ }^{1}$ : outre les articles écrits à vif, au Mexique, juste après son Voyage au Pays des Tarahumaras, en septembre-octobre 1936, tels "La montagne des Signes", "Le pays des Rois Mages", "Une Race-Principe", etc, une importance particulière sera donnée à l'écriture du dernier Artaud, tels les deux poèmes "Tutugurin, écrits l'un en octobre 1947, l'autre le 16 février 1948, quelques jours avant sa mort. Cependant, ce sont les textes consacrés aux rites du Ciguri, tels "La danse du Peyotl" récrit en 1937, ou "Le Rite du Peyotl chez les Tarahumaras", écrit à Rodez en 1943, puis remanié en 1947 lors du Retour à Paris, qui seront en quelque sorte le catalyseur du montage des images, des sons et de la voix du texte, son fil directeur: l'Idée du film, si l'on veut.

Car l'intérêt de ces textes d'Artaud, la fascination qu'ils exercent sur nous, si on les considère dans leur processus de création, est bien l'impossibilité essentielle d'écrire les sensations et les émotions mêmes du Ciguri, leur dimension fondamentale. Comme si les descriptions de la Montagne des Signes, ou la relation de la Danse du Peyotl étaient encore trop extérieures, manquaient radicalement cet invisible qui est le plus important de l'initiation:

1 On sait que le texte "définitif" établi par Artaud avant sa mort avec son éditeur Marc Barbezat rassemblait sous le titre Les Tarabumaras la plupart des textes déjà publiés dès 1937 par Jean Paulhan sous le titre "D'un Voyage au Pays des Tarahumaras" et les faisait précéder par le texte "Le Rite du Peyotl chez les Tarahumaras". Le poème "Tutuguri" était placé après "D'un Voyage...", si bien que le corps du livre Les Tarahumaras se présente essentiellement comme une sorte de triptyque, de composition en trois volets, selon l'ordre: 1. "Le Rite du Peyotl..." 2. "D'un Voyage..." 3. "Tutugurin. (Voir sur ce point les notes critiques de Paule Thévenin, Antonin ARTAuD, Oeuvres complètes (Paris: Gallimard), tome IX. 
Couché bas, pour que tombe sur moi le rite, pour que le feu, les chants, les cris, la danse et la nuit même, comme une voûte animée, humaine, tourne vivante, au-dessus de moi. Il y avait donc cette voûte roulante, cet agencement matériel, de cris, d'accents, de pas, de chants. Mais, par dessus tout, au delà de tout, cette impression, qui revenait, que derrière tout cela, plus que tout cela, et au-delà, se dissimulait encore autre chose: le Principal.

Comme si le récit des étapes du rite restait encore sur le plan de la conscience ordinaire, de ses rêves, de ses fantasmes, celui de "l'irréalité, de l'illusion, du non-fait, du non-préparé.. Comme s'il fallait gagner, par l'écriture elle-même, cet autre plan auquel nous fait accéder Ciguri: "un plan où la conscience normale n'atteint pas mais où Ciguri nous permet d'atteindre, et qui est le mystère même de toute poésiem. Il y aurait donc nécessité absolue de ré-écrire l'impression première, toujours après-coup, puisque l'écriture immédiate en est d'emblée interdite, vouée à inachèvement.

C'est cette singularité d'un impouvoir d'écriture propre à Ciguri qui est marquée par Artaud dès son retour à Paris, début 1937, lorsqu'il parle de récrire "La Danse du Peyotl", texte qu'il a commencé à la fin de son séjour au Mexique, et qu'il lui "a été impossible d'achever. Il s'obstinera à briser cet inachèvement, s'acharnera à vaincre les résistances spécifiques à cette écriture, et remettra le texte à Jean Paulhan, en deux fois: la première partie le 13 mars, et "enfin" la seconde, le 28 mars 1937. La dernière page de "La Danse du Peyotl", très belle, dit dans une sorte d'abrupt le doute fondamental d'Artaud quant à la réussite de son entreprise, quant à la possibilité d'écrire "le secret", "le mystère", "l'être invisible" de Ciguri:

Je n'avais pas été cet homme de pierre auquel il fallait deux hommes pour en faire un homme monté: et qu'on montait et descendait de cheval comme un automate désemparé [...]; je n'avais pas vaincu à force d'esprit cette invincible hostilité organique, où c'était moi qui ne voulais plus marcher, pour en ramener une collection d'imageries périmées [...]. Il fallait désormais que le quelque chose d'enfoui derrière cette trituration pesante et qui égalise l'aube à la nuit, ce quelque chose fût tiré dehors, et qu'il servit...

Ce n'est donc pas un hasard si le texte de 1943 "Le Rite du Peyotl chez les Tarahumaras" ré-écrit autrement l'initiation initiale, en réinterroge à nouveau "le mystère", puis, lors du Retour à Paris, remanie en 1947 le texte de Rodez, en supprime toute trace de conversion à Jésus Christ, tente une dernière fois d'extraire l'invisible païen des rites du Ciguri. C'est ce texte enfin rendu à la vérité du Ciguri qui ouvre Les Tarabumaras à la demande expresse d'Artaud à son éditeur en juin 1947, rendant visible par la composition même du livre un ordre plus essentiel que celui de 
l'exposition chronologique, encadrant les textes "D'un Voyage au Pays des Tarahumaras" par les textes dits du Retour à Paris, ceux de "la Grande Santé,, selon l'expression de Nietzsche.

Ainsi, la lecture des Oeuvres complètes d'Antonin Artaud, aujourd'hui (presque) possible, notre propre recherche cinématographique de dix ans (1977-87) chez les Indiens Tarahumaras de Norogachic, seul lieu de la Sierra explicitement nommé par Artaud, nous persuadent de l'importance fondamentale de ce que nous appelons l'Expérience Tarahumara dans l'oeuvre d'Artaud, indissolublement écriture et vie, poésie et pensée. Experience dont Ciguri est le centre -entendons ici par Ciguri non seulement l'initiation d'Artaud au rite du peyotl, mais la lettre même du mot, jusque dans sa décomposition sonore en voyelles et consonnes, l'événement d'écriture, à la fois corps et pensée que ce nom provoque, comme un prénom singulier.

A un moment quelque chose comme un vent se leva et les espaces reculèrent. Du côté où était ma rate un vide immense se creusa qui se peignit en gris et rose comme la rive de la mer. Et au fond de ce vide apparut la forme d'une racine échouée, une sorte de $\mathrm{J}$ qui aurait eu à son sommet trois branches surmontées d'un E triste et brillant comme un oeil. Des flammes sortirent de l'oreille gauche de J... En tout cas j'avais vu, paraît-il, l'Esprit même de Ciguri.

Car Ciguri n'est pas seulement l'ancien rite d'Indiens perdus dans la montagne, mais la recherche actuelle d'un autre corps, "corps sans organes", seulement fait d'os et de sang, l'acte par lequel les forces de la vie se rassemblent pour se refaire un corps: ale corps/rien que le corps/seul le corps".

Par cet acte, corps et écriture, voix et poésie se construisent d'un seul tenant, sur un seul et même plan. Car seule une voix, les rythmes et les scansions propres d'un corps, peuvent libérer les forces du texte d'Artaud, réveiller les puissances de l'image-cinéma, arallumer encore en nous, en chacun de nous, de rapaces besoins d'envol. Car le cinéma est aussi notre mythe ${ }^{2}$.

Il s'agit donc, au plus loin d'un documentaire ethnographique comme d'un document d'histoire littéraire, de confronter par le montage: 1. les images et les sons des pratiques rituelles les plus sacrées de ceux qu'Artaud nommait "une Race-Principe" (de ce que nous pourrons en filmer, "car le danger des mythes, si hauts soient-ils, c'est qu'ils s'éteignent"); 2. les images et les sons de ces paysages de hauts plateaux désertiques, peu-

2 "Et les Mythes au Mexique, pour peu qu'on y regarde, rallument encore en nous, en chacun de nous, de rapaces besoins d'envolw. Antonin ARTAUD, Oeuvres complètes, tome V, p. 161. 
plés de Silence, où se découvre d'un coup l'horizon infini des lignes bleues des montagnes, la superposition des plans dans la transparence de l'air, comme les "Sainte Victoire" de Cézanne; 3. la voix de grands acteurs (Samy Frey ou David Warrilow/Maria de Medeiros...), lisant les textes Tarahumaras d'Artaud, enfin.

Finalement, les rencontres et les écarts, les intersections et les décalages, les accords et désaccords entre ces différents éléments visuels et sonores doivent donner à voir quelque chose du combat entrepris par Artaud dans son Expérience Tarahumara, et qu'il ne cessera de soutenir jusqu'à la fin de sa vie: la recherche d'une forme nouvelle où l'Art et le Sacré, poésie et vie, Ciel et Terre, Espace et Corps communiquent dans la violence d'une brisure, la fulgurance même du Feu, ule spasme de l'être": moment où "le dieu-et-homme s'accouple", comme disait Hölderlin, non dans l'apaisement des contraires, mais dans "un détournement catégorique", "une séparation et un atermoiement illimités". Il est significatif ici qu'Artaud, découvrant dans le Rite du Peyotl la danse des deux servants du prêtre du Ciguri, l'homme et la femme, emploie pour dire leur accouplement cosmique des termes à la fois absolument singuliers et au plus proche de ceux d'Hölderlin:

Le prêtre alors cracha: non pas de la salive mais son souffle. Il expulsa bruyamment son souffle entre ses dents. Et sous l'action de cet ébranlement pulmonaire l'homme et la femme au même instant s'animèrent et se levèrent complètement. Or, à la façon dont ils se tenaient l'un devant l'autre, à la façon surtout dont ils se tenaient chacun dans l'espace comme ils se seraient tenus dans les poches du vide et les coupures de l'infini on comprenait que ce n'était plus du tout un homme et une femme qui étaient là mais deux principes: le mâle, bouche ouverte, aux gencives claquantes, rouges, embrasées, sanglantes, et comme déchiquetées par les racines des dents, translucides à ce moment-là, telles des langues de commandement; la femelle, larve édentée, aux molaires trouées par la lime, comme une rate dans sa ratière, comprimée dans son propre rut, fuyant, tournant devant le mâle hirsute; et qu'ils allaient s'entre-heurter, s'enfoncer frénétiquement l'un dans l'autre comme des choses, après s'être regardées un temps et fait la guerre, s'entremêlent finalement devant l'oeil indiscret et coupable de Dieu, que leur action doit peu à peu supplanter ${ }^{3}$.

3 Antonin ARTAUD, "Le rite du peyotl chez les Tarahumaras", Oeuvres complètes, tome IX, pp. 26-27. On jugera de la proximité de l'expérience Tarahumara d'Artaud avec la pensée du Tragique chez Hölderlin, en confrontant le texte cité ici avec ce fragment des Remarques sur Oedipe: aLa présentation du tragique repose principalement sur ceci que l'insoutenable, comment le Dieu-et-homme s'accouple, et comment, toute limite abolie, la puissance panique de la nature et le tréfonds de l'homme deviennent Un dans la fureur, se conçoit par ceci que le devenir-un illimité se purifie par une séparation illimitée". 
Car, malgré cette proximité, l'extrême modernité d'Artaud (son actualité, en un mot) est la lutte incessante pour éliminer le tragique de la séparation, en finir avec une pensée de l'écriture et du sacré toujours soumise au jugement de Dieu. Dans ce combat pour l'homme, un corps humain, seulement humain, la seule arme et la seule stratégie d'Artaud sont l'humour et le rire.

En tout cas j'avais vu, paraît-il, l'Esprit même de Ciguri [...] et les Mystiques doivent passer par des états et des images pareilles avant d'atteindre suivant la formule aux suprêmes embrassements et déchirements, après lesquels ils tombent sous le - baiser de Dieu comme des poules sans doute dans les bras de leur maquereau ${ }^{4}$.

C'est cette légèreté de l'humour, cette lucidité du rire d'Artaud qui doivent être la grande santé du film, son ton fondamental.

\section{FORME DU FILM: LA ROUTE DU CIGURI}

La question de l'élaboration du film, de sa construction, supposent donc une sorte d'écriture-montage-fiction, scénario préalable qui sera le véritable schème du film, le principe virtuel de son processus de création: de son tournage comme de son montage, de son rythme: la recherche de la forme.

Nous ne voyons pas d'autre chemin que celui de répéter, à notre tour, bien plus modeste et cependant périlleux, avec les matériaux spécifiques de l'image et du son, l'Expérience Tarahumara d'Antonin Artaud: ré-écrire aujourd'hui, cinématographiquement et donc autrement, le processus d'écriture poétique d'Artaud, dans un mouvement interminable, incessant (celui de toute écriture, de toute lecture). Il y aura donc un double mouvement du film, un double récit, un autre film doublant comme souterrainement le film: la répétition actuelle D'un Voyage au Pays des Tarahumaras, où le récit de la quête cinématographiquc de Ciguri, aujourd'hui, redouble et dédouble l'expérience d'Artaud. D'où le pluriel de notre titre Voyage(s)... disséminant ces jeux du double entre hier et aujourd'hui, passé et présent, vie et écriture, cinéma et poésie: 1936/1947 quant à Artaud; 193647/1994 quant à une lecture d'Artaud, aujourd'hui; 1977-87/1994-95 quant à notre propre expérience cinématographique chez les Tarahumaras.

On reprendra donc la forme de récit créée par Artaud pour dire son expérience du Ciguri, celle qu'il trouve d'un coup dans sa première manière de récrire "La Danse du Peyotl" en 1937, inventant une sorte de

\footnotetext{
4 Antonin ARTAUd, "Le rite du peyotl chez les Tarahumaras", op. cit., p. 36.
} 
Nouvelle poétique entre le journal de voyage inspiré et la rigueur extrême de la pure poésie; forme qu'il ré-élaborera dans un statut calculé, au cours des deux versions du "Rite du Peyotl..." $(1943,1947)$, alternant cette fois la lenteur de la pensée qui cherche à extraire le sens de l'expérience initiale et les vitesses de scènes fulgurantes, aux images visionnaires, marquant ainsi le suspens des étapes de l'initiation dans des césures savantes, trouvant le rythme propre du combat gigantesque de l'écriture entre poésie et pensée: une véritable mécanè, au sens de Hölderlin 5.

On re-commencera donc la route du Ciguri, le récit-fiction de cette initiation en trois temps, selon Antonin Artaud, où "le glaive du Maître de toutes les choses", puis "l'onction des prêtres du soleil (Tutuguri)" constituent les premiers degrés de "la route du Tutuguri", avant "la frappe immersive et réagrégatrice des prêtres du Ciguri, qui est un rite d'anéantissement": avant l'étape ultime de "la route du Ciguri».

Comme je l'ai déjà dit, ce sont les prêtres du Tutuguri qui m’ont ouvert la route du Ciguri comme quelques jours auparavant le Maître de toutes les choses m'avait ouvert la route du Tutuguri. Le Maître de toutes les choses est celui qui commande aux relations extérieures entre les hommes: l'amitié, la pitié, l'aumône, la fidélité, la piété, la générosité, le travail. [...] Nul au Mexique ne peut être initié, c'est-à-dire recevoir l'onction des prêtres du Soleil et la frappe immersive et réagrégatrice de ceux du Ciguri, qui est un rite d'anéantissement, s'il n'a été auparavant touché par le glaive du vieux chef indien qui commande à la paix et à la guerre, à la Justice, au Mariage et à l'Amour.

La route du Tutuguri sera rapidement évoquée, à travers quelques images visionnaires des rites du Soleil, mais c'est la route du Ciguri qui sera l'objet de la quête cinématographique, comme si le film Ciguri était lui-même un rite d'initiation, comportant les épreuves, le suspens, les éblouissements d'un chemin périlleux.

La difficulté de l'approche de ces rites jusqu'ici tenus secrets, le caractère probablement partiel de ce que les prêtres du peyotl nous autoriseront à filmer, les risques du tournage enfin, nous placent devant la nécessité d'inventer, de créer un type de plans qui donne à voir et à imaginer: une mise en images du réel traversée par ces puissances fulgurantes du Dehors qui ne cessent de hanter l'écriture d'Artaud. Le film alternera donc à son tour les moments de vision poétique (images-paysages, gros plans-visage, images-intensités extraites des Rites du Tutuguri et du Ciguri) et les moments plus proches de la réalité ethnographique

5 Dans les Remarques sur Oedipe, Hölderlin définit la mécanè comme ale calcul du statut de l'oeuvre et les démarches méthodiques grâce auxquelles le beau est produit". 
d'aujourd'hui, où la voix du texte d'Artaud cherchera le sens perdu de ces rites.

Dans les moments de travail de la pensée, la voix pourra être dite voix de lecture intérieure, proche l'émergence de l'écriture: voix neutre, elle-même en recherche, laissant sourdre les mots, avant même l'affleurement du sens. Dans les moments où les images vivantes jaillissent librement, la voix pourra laisser passer l'émotion, dire la vision, se ralentir ou se précipiter dans des mots-souffles et des mots-cris.

Cependant, rien ne sera systématique, au contraire. Là encore, une recherche est à faire. Et d'abord, la distinction proposée ici entre lire et dire: voix du texte lu et du texte dit, est-elle pertinente? Nous le croyons. Le travail de l'acteur David Warrilow à partir des textes de Beckett ou de Pinget est pour nous exemplaire d'un passage possible entre la voix de lecture intérieure retenant le sens au dedans, avant-naissance et la voix émotionnelle sortant le texte au dehors: hors de soi et cependant comme rejeté vers l'intérieur ${ }^{6}$.

C'est le travail avec les acteurs comme celui du montage audio-visuel, la confrontation des images et des sons, qui décideront. Pour l'heure, contentons-nous de poser la question de la possibilité d'une variation continue entre ces deux voix.

Le Hors cadre de la vie, du corps, de la poésie, doit traverser le cadre de l'image, comme les deux poèmes "Tutuguri", en 1947 et 1948, réécrivent l'expérience du Voyage au pays des Tarahumaras de 1936, découvrent par la voix, "les voyelles chantées", le cri, les sons et les rythmes, la dimension, cosmique, des Rites Tarahumaras ${ }^{7}$.

6 "Là où l'athlète s'appuie pour courir, c'est là que l'acteur s'appuie pour lancer une imprécation spasmodique, mais dont la course est rejetée vers l'intérieur". Antonin ARTAuD, "Un athlète affectif", Oeuvres complètes, tome IV, p. 155. Or, ce travail de la voix de l'acteur est le travail même du corps et de la pensée dans l'expérience du Ciguri: "L'emprise physique était toujours là. Ce cataclysme qui était mon corps... Après vingt-huit jours d'attente, je n'étais pas encore rentré en moi; -il faudrait dire: sorti en moi. En moi, dans cet assemblage disloqué, ce morceau de géologie avariée». "La Danse du peyotl", Oeuvres complètes, tome IX, p. 48.

7 Artaud a inséré "Tutuguri, le rite du soleil noir" dans l'émission radiophonique "Pour en finir avec le jugement de Dieu. Il fut lu par María Casares. Il fait donc partie du texte "Pour en finir..... Le second "Tutuguri", réécrit pour le livre Les Tarabumaras, est plus long, "lourd d'une sanglante expérience que je n'avais pas en 1936. Cette sanglante expérience est que je viens d'avoir trois attaques où l'on m'a trouvé baignant dans mon sang..... Cette seconde version poursuit l'expérimentation sonore commencée en 1947, introduit à l'intérieur même du poème le battement du rythme de la voix, le tympanon de bois du Tutuguri, comme les instruments cosmiques des éléments: «le bruit des grandes cloches au vent, le déchirement des canons de la marine, l'aboi des vagues dans les tempêtes des autans". Le poème se termine 
C'est cette dimension nouvelle de notre recherche Tarahumara que nous devons gagner dans ce film Ciguri, après une interruption de sept ans. De cette expérience de création rien ne sera dit, mais seulement montré à travers le chemin anonyme, à la fois singulier et pré-personnel d'un récit valable pour tous, "pour chacun de nous".

Pour trouver la forme cinématographique de ce récit nous proposons un type d'écriture, fragmentaire, montage d'extraits-citations des textes Tarahumaras d'Antonin Artaud et des images du film à venir. Cette écriture est aussi exigeante et rigoureuse que celle d'un scénario de fiction proprement dit: outre la recherche de fragments disséminés dans l'oeuvre entière d'Artaud, outre leur mise en ordre selon ce qu'on peut appeler la logique du Ciguri, il s'agit de donner à voir et à entendre ce centre (invisible) de l'expérience d'Artaud.

El ensayo presenta la problemática que significa la realización de un film etnográfico $y$, específicamente, la construcción de un discurso fílmico propio, por lo que se propone que es necesaria la construcción de un "escenario" ideacional que permita captar los múltiples detalles de la realidad que se quiere filmar. El artículo se organiza a partir de un proyecto singular, a caballo entre la literatura y el cine, entre el cine y la etnología, que ha consistido en traducir en imágenes el Voyage que Antonin Artaud hizo a México en 1936, en concreto, al país de los Tarahumaras.

The author discusses the problems involved in ethnographic film-making, specifically in the making of a film discourse of one's own. She deems it necessary to construct an ideational "scenario" in which to capture the multiple details of the reality that is to be filmed. The visual representation of Antonin Artaud's Voyage of 1936 to Tarahumara territory, in Mexico, is a case in point: a unique project which combines literature and film-making, film-making and ethnology.

sur l'image de l'homme-cheval, du tronc de l'homme "pris à la balafre de son sang". Ce sont ces images déjà audio-visuelles que le film doit laisser passer. (Nous choisirons cette seconde version, dans des images qui devraient être le finale du film, suivant en cela l'ordre de composition du livre Les Tarahumaras). 